\title{
Detection and tracking of clathrin-coated pits in biological images
}

\author{
LIU ZhiFeng ${ }^{1}$, GE Yun ${ }^{1 *}$, ZHANG Dong ${ }^{1} \&$ ZHOU XiaoBo ${ }^{2}$ \\ ${ }^{1}$ School of Electronic Science and Engineering, Nanjing University, Nanjing 210093, China; \\ ${ }^{2}$ HCNR-Center for Bioinformatics, Harvard Medical School, MA 02215, USA
}

Received August 15, 2011; accepted September 26, 2011

\begin{abstract}
Dynamically tracking hundreds of individual pits is essential to determine whether there exist "hot spots" for the formation of clathrin-coated pits or if the pits formed randomly on the plasma membrane. We propose an automated approach to detect these particles based on an improved á trous wavelet transform decomposition with automatic threshold selection and post processing solution, and to track the dynamic process with a greedy algorithm. The results indicate that the detection method can successfully detect most particles in an image with accuracy of $98.61 \%$ and $97.65 \%$ for adaptor and clathrin images, respectively, and that the tracking algorithm can resolve merging and splitting issues encountered when analyzing dynamic, live-cell images of clathrin assemblies.
\end{abstract}

particle detection, particle tracking, wavelet transform, greedy algorithm

Citation: Liu Z F, Ge Y, Zhang D, et al. Detection and tracking of clathrin-coated pits in biological images. Chin Sci Bull, 2012, 57: 729-735, doi: $10.1007 / \mathrm{s} 11434-011-4895-\mathrm{Z}$

Recently, much attention has been focused on the development of segmentation and particle tracking techniques for the analysis of large volumes of microscopy fluorescence images acquired during cellular level cell imaging $[1,2]$. Particle tracking is one method of acquiring information about cellular dynamics. Clathrin-coated pits can be found in all nucleated cells, and provide an important means by which proteins and lipids are removed from the plasma membrane (endocytosis) and transported to an internal compartment (endosome) [3]. Using electronic microscopes, fluorescent-labeled versions of a variety of marker proteins have provided a tantalizing glimpse into the dynamics of the system in living cells. The clathrin pathway has thus acquired special status for analyzing molecular mechanisms in membrane traffic. A single image may capture hundreds or thousands of coated pits forming at the surface of a cell, all of which may not behave in the same way in time and space. Thus, information about these objects may need to be extracted and assembled at different time points and correlated with information from other images obtained at different

*Corresponding author (email: geyun@nju.edu.cn) spectral frequencies. For that purpose, clathrin light chains can be tagged with fluorescent proteins such as enhanced green fluorescent protein (EGFP) or yellow fluorescent protein (YFP) [1], and long time-series data can then be acquired using wide-field, confocal and total internal reflection fluorescence (TIRF) microscopy. Owing to the large quantity of images and hundreds of coated pits in each obtained image, an automated analysis approach is therefore necessary to visualize such molecular imaging data.

The process for computerized analysis of cellular microscopy images generally consists of auto-segmentation, tracking and feature extraction. The detection of particles is the most critical step in molecular or sub-cellular image analysis where knowledge of the morphology of particles and the distribution of fluorescence signals in the particles is required. Although several particle detection methods based on segmentation have been developed [4,5], results have varied. Under-segmentation and/or over-segmentation can occur because these methods are based only on the intensity of the images or the morphological shape. A multiscale wavelet products method has been successfully applied to extract particles with arbitrary shape and size from 
a single image with an inhomogeneous gray level distribution of both background and particles $[1,6]$. However, it cannot be used directly in segmenting image series with a fixed threshold because of the image intensity variance over the entire image series.

Particle tracking is concerned with the tracking of multiple moving objects in an image sequence and reconstruction of all of the trajectories of these objects over the entire sequence [7]. Recently, several algorithms have been developed for tracking the motion of particles and cells [8-12]. One method is based on appearance features [11], which assume that the shape of a certain cell or particle changes only slightly between consecutive frames. Another method is to maximize the smoothness of the particle trajectory and the velocity $[10,12]$ for the trajectory direction and the velocity of a moving particle should only change slightly between consecutive frames. However, these methods do not work well with our data set as only partial information of the particles' movement is considered. Sethi and Jain [13] proposed a tracking algorithm based on the greedy principle and smoothing criteria with the constraint of a constant number of particles in all the frames. Furthermore, Chetverikov and Verestóy [14] studied particle track creation and termination based on the cost function of the algorithm proposed by Sethi and Jain. However, the particle splitting and merging scenario was not considered in either study.

This study considers the detection and tracking problem described above. First, we apply an adaptive threshold based on the entropy of the image histogram and a post-processing solution to segment the image series. Second, we propose a new N-to-N correspondence tracking approach based on the stable match and greedy algorithm. The proposed method aims to address the problems of trajectory creation, splitting, merging, and termination.

\section{Particle detection using a multi-scale product approach}

The task of automated spot tracking in video sequences is particularly challenging because of the spots' indistinct shapes, the potential instability of the video, an abundance of moving objects, and their frequent superposition. To address any of these problems, one must first detect spots accurately. An algorithm based on the 'á trous' wavelet transform has been successfully developed for the detection of fluorescent spots in microscopy images [6]. Since this approach is effective in detecting different sizes of particles in one image, here we extend it to the detection of clathrin particles combined with local information such as area, intensity and shape.

The á trous wavelet decomposition is a translation-invariant, non-decimated wavelet transformation based on successive convolutions with B-spline based kernels. The original wavelet filter $H_{0}$ is convoluted row by row and column by column with the kernel $[1 / 16,1 / 4,3 / 8,3 / 8,1 / 4,1 / 16]$
[15]. The smooth image $A_{1}(x, y)$ is computed as the convolution of original image $A_{0}(x, y)$ and filter $H_{0}$. The first wavelet layer $W_{1}(x, y)=A_{0}(x, y)-A_{1}(x, y)$ is the difference image of the original image and the filtered image. Other wavelet layers $W_{i}(x, y)$ can be obtained by repeating this process:

$$
W_{i}(x, y)=A_{i-1}(x, y)-A_{i}(x, y) \quad(1 \leqslant i \leqslant 2)
$$

where $A_{i}(x, y)=A_{i-1}(x, y) \otimes H_{i}(x, y), A_{i}(x, y)$ is the $i$ th layer of the smoothed image, and $A_{i-1}(x, y)$ is the $(i-1)$ th layer of the smoothed image. $H_{i}$ is calculated by inserting $2^{i-1}-1$ zeros between two taps of $H_{0}$. Reducing the noise of each of the coefficient layers is performed by applying a threshold with a median absolute deviation. Then, all coefficient layers are multiplied to generate the multi-scale product images.

A threshold based on the entropy of histogram is applied to the multi-scale product images, which is implemented by minimizing the following equation [16]:

$$
\begin{aligned}
f(k)= & P(T) \log \sigma_{b}(T)+[1-P(T)] \log \sigma_{f}(T) \\
& -P(T) \log P(T)-[1-P(T)] \log [1-P(T)] .
\end{aligned}
$$

Image pixels are then divided into two sets, i.e. background $(1, \ldots k)$ and particle object $(k+1, \ldots 255)$, and $P(T)$ gives the estimate of the probability of occurrence of the background pixels; $\sigma_{b}, \sigma_{f}$ are the standard deviation of the background and particle object respectively. The optimized threshold $k$ is solved by

$$
k=\arg \min f(k)
$$

The obtained threshold $k$ is used to binarize the products images and extract all the particles. We then apply the signal-noise ratio (SNR) method to eliminate the weak spots detected using the above proposed detection method. The basic idea of the SNR method is that a spot will be eliminated if the SNR of the spot is too low. Global SNR is calculated from the difference in mean intensity between the object $I_{s}$ and background $I_{b}$, divided by a representative noise level as

$$
\mathrm{SNR}=\left(I_{s}-I_{b}\right) / \sigma_{b},
$$

where $I_{s}$ is the average intensity of the detected particles, $I_{b}$ is the average intensity of the background pixels, and $\sigma_{b}$ is the standard deviation of the background.

To estimate the local SNR for each particle, we choose a circular region with the center locating at the centroid of the particle, and the diameter as ten pixels. Then, we denote $I_{s p}$ as the average of the detected signals, $I_{b p}$ as the average of the detected background, and $\sigma_{b p}$ as the standard deviation of the background of this region. The local SNR for spot $P$ is computed by

$$
\mathrm{SNR}_{p}=\left(I_{s p}-I_{b p}\right) / \sigma_{b p} .
$$

Total intensity is calculated by 


$$
I_{p}=\left(I_{s p}-I_{b p}\right) \times S,
$$

where $S$ is the area of the particle.

\section{Particle tracking using the greedy algorithm}

After particles are detected in all relevant frames, particles can be tracked by a motion correspondence algorithm [17]. If small particles are moving individually and independently, several single-particle tracking methods can be used [18]. However, these methods are not applicable in some situations. For example, particles may approach one another at distances that can no longer be distinguished so that they merge into one single spot. Or, conversely, a large spot that seems to be a single particle in one frame may split into several small particles in the next frame. The main goal of the tracking method presented here is to solve the ambiguous correspondence problem in particle movement.

In our algorithm, the detected particles are used as inputs, and their projections on different frames are connected into trajectories. The matrix of frame $k$ is defined as

$$
A_{k}=a_{i j}^{k}, 1 \leqslant i \leqslant m, 1 \leqslant j \leqslant n,
$$

where $k$ is the layer number of the processed frames, $m$ is the total number of trajectories in the frame $k, n$ is the total number of particles detected from the frame $k+1, i$ is the number of the trajectory in frame $k$, and $j$ is the number of the particle in frame $k+1$. When $a_{i j}^{k}=0$, no relation exists between the particle $j$ and track $i$; when $a_{i j}^{k}=1$, the particle $j$ only belongs to the track $i$. Thus, track splitting occurs while $\sum_{j=1}^{n} a_{i j}^{k}>1$, and track merging occurs while $\sum_{i=1}^{m} a_{i j}^{k}>1$.

The splitting or merging of particles may result from a biological phenomenon or from the projection of 3D particle onto 2D frames during image acquisition. Automatic processing of splitting and merging can improve the tracking efficiency. We apply the idea of a stable match in two adjacent frames to solve this problem. If one point of any pair in the match has a better correspondence choice, this pair is recognized as unstable. The matching set is considered as stable only if no unstable pair exists [7].

Match selection based on the smooth rule uses a cost function defined by the following equation [16], which is related with three layers:

$$
\delta\left(P_{k-1, q}, P_{k, r}, P_{k+1, s}\right)=\omega_{1} \eta_{1}+\omega_{2} \eta_{2},
$$

where $P_{k-1, q}$ is the $q$ th particle in the frame $k-1, P_{k, r}$ is the $r$ th particle in the frame $k$, and $P_{k+1, s}$ is the sth particle in the frame $k+1$.

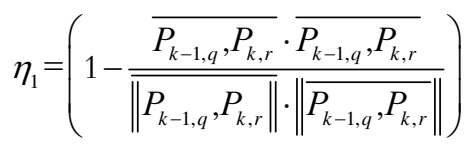

describes the changes in direction.

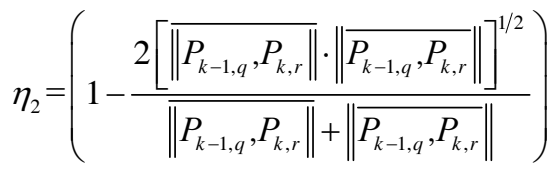

represents the variance of the velocity. $\omega_{1}$ and $\omega_{2}$ are the weights of $\eta_{1}$ and $\eta_{2}$ respectively, and satisfy $\omega_{1}+\omega_{2}=1$.

It is necessary to initialize the correspondence matrix $A_{1}$ for the first two frames using the nearest neighbor criteria, as the velocity and direction used in the tracking process are determined by more than two frames. Using the maximum displacement constraint $\phi_{\max }$ as the radius, we can determine the searching area for $P_{1, i}$ in the second frame, and find all possible candidate particles. Every possible candidate pair is chosen, and its cost is calculated by $\delta_{i j}=\left(x_{1, i}-x_{2, j}\right)^{2}+\left(y_{1, i}-y_{2, j}\right)^{2}$, where $x_{1, i}, y_{1, i}$ are the $x$-axial and $y$-axial values of the $i$ th particle in the first frame, respectively, and $x_{2, j}, y_{2, j}$ are the $x$-axial and $y$-axial values of the $j$ th particle in the second frame, respectively. The matching pairs are then sorted incrementally by cost. The pair with the lowest cost in the pair candidates will be added into the correspondence matrix $A_{1}$. The relationship between the particles in the first two frames is built by repeating this process. Those particles without associations in the second frame are considered to be the start of a new track; otherwise if they are in the first frame, they are viewed as the end of a new track.

The merging process finds an N-1 correspondence relationship between the frame $k$ and frame $k+1$, while the splitting process is the inverse relationship. The tracking method is named as the admission algorithm, and is performed in the forward direction to address the merging problem and in the backward direction to solve the splitting problem. An overview of the splitting and merging process is shown in Figure 1.

The merging process is performed using the following three steps: (1) Find the particle candidates in the frame $k+1$ that satisfy the maximum displacement constraint $\phi_{\max }$ for each particle $P_{k, i}$ in the frame $k$, and store all candidates sorted incrementally by $\operatorname{cost} \delta$. (2) Every particle $P_{k, i}$ is applied to its best correspondent, and the relationship is built; $P_{k+1, j}$ realigns all the applicants incrementally by cost, and picks up one or more applicants and rejects others. The selecting rule satisfies the total net fluorescence invariant cri-

teria: $\left|\frac{A_{\alpha}(t+1)}{\sum_{i=1}^{m} A_{i}(t)}-1\right|<$ thre, where $A_{\alpha}(t+1)$ is the total net fluorescence of the merged particle, and $A_{i}(t)$ indicates the total net fluorescence of each child particle. The total net fluorescence for a given particle is its fluorescence intensity minus the fluorescence intensity of the background of an area with the same size. (3) The rejected particle will remove its best candidate and apply to its next preferred candidate, if it 


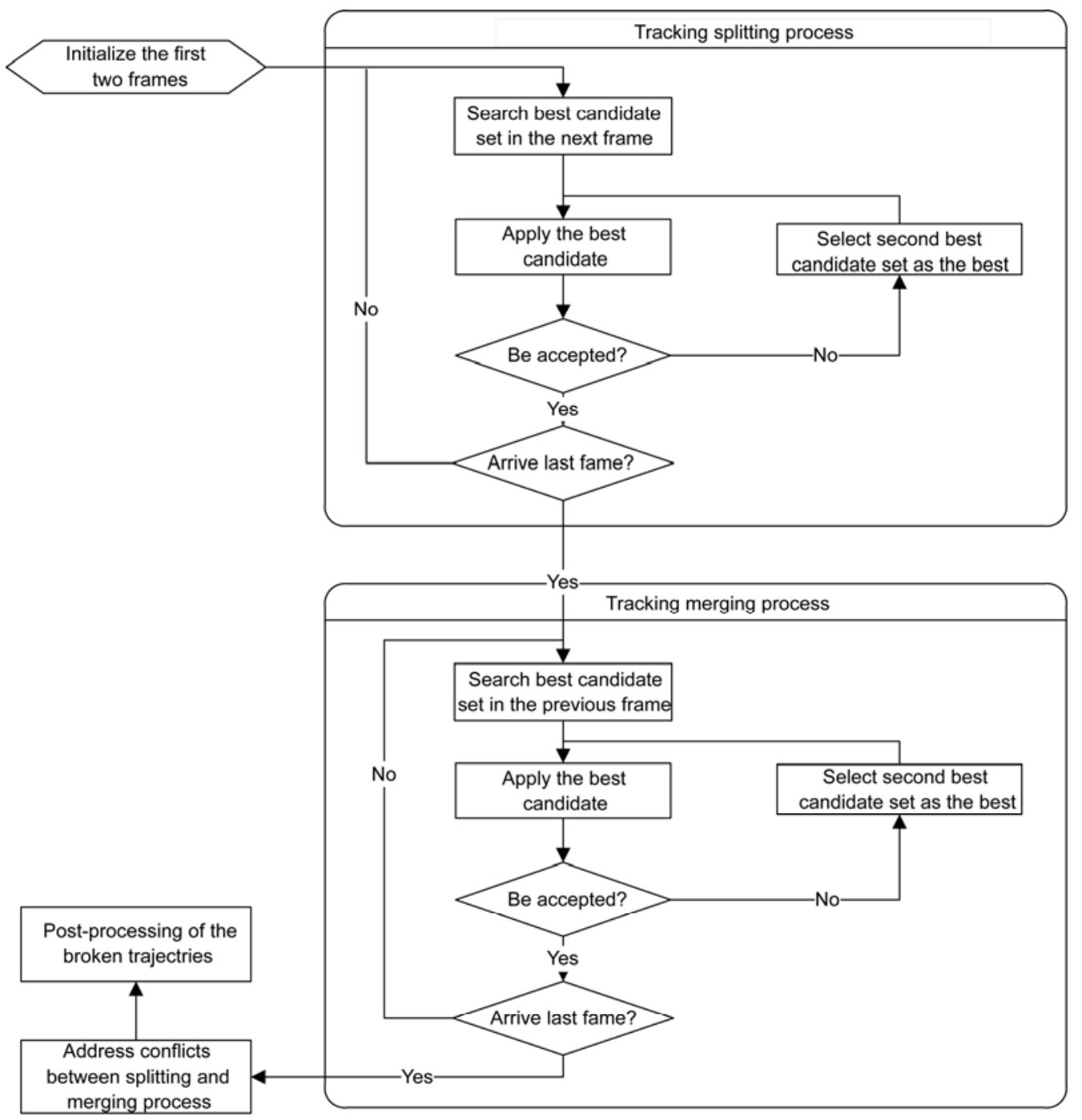

Figure 1 Overview of the splitting and merging process.

exists. If any submission or rejection occurs, the algorithm moves to step (2) to repeat the selection process; otherwise, the iteration process ends and the generation of the association matrix $A_{k}^{\text {merge }}$ is finished. The splitting process is the reverse version of the merging process. It is reversed by switching the two frames, and performing the process again, by which we can get the association matrix $A_{k}^{\text {split }}$ which handles the splitting problem.

To combine the merging and splitting parts into an association, two matrixes $A_{k}^{\text {merge }}$ and $A_{k}^{\text {split }}$ are incorporated together. If one particle merges and splits at the same time, a conflict will occur. These conflicts are solved by means of the following rules: (1) If a small child particle has its own children particles, it will be discarded by its parent particle; (2) if conflicts exist while no merging/splitting happens, the association pair with the higher cost is discarded.

This process is repeated until the last frame, when all of the trajectories will be totally extracted. Since images are acquired in two dimensions, some particles may be temporarily out of the focal plane of the microscopy imaging system. Shaw and Ramachandran [19] indicated that this filling-in occurs unless at least two frames on either side of the frame with the missing points are available. The occluded particle in frame $k$ is interpolated using the previous and subsequent two frames, and then the two broken trajectories are linked together.

\section{Experimental results}

Images were acquired with a spinning disk confocal coupled to a fully motorized epifluorescence microscope using $63 \times$ or $100 \times$ lenses under control of SlideBook software. 12-bit digital images were obtained with a cooled CCD camera with $2 \times 2$ binning and a spatial resolution of 140 or 200 $\mathrm{nm} /$ pixel for the two lenses, respectively. In our study, the acquired 12-bit digital images were transformed into 8-bit 
images to reduce computation costs. Images were acquired with exposure times between 800 and $1200 \mathrm{~ms}$ from cells maintained at $37^{\circ} \mathrm{C}$ by using a heated stage [1]. We studied adaptor and clathrin movies (available in the corresponding Cell website) to show that the proposed approach was effective for automatic particle segmentation and tracking. In the first movie, adaptor was labeled by LCa-YFP, having 120 frames with intervals of $10 \mathrm{~s}$; while clathrin was labeled by LCa-EGFP, having 200 frames with intervals of $3 \mathrm{~s}$. We first address the accuracy of particle detection, followed by the accuracy of the particle tracking.

Figures 2 and 3 show the detection results for the adaptor and clathrin images respectively, where the decomposition layer is 2. Figures 2(a) and 3(a) are original images of the adaptor and clathrin, respectively. Figures 2(b) and 3(b) present the detection results using the improved multi-scale product wavelet method.

To evaluate the accuracy of particle detection, we define four parameters to demonstrate the performance of our detection method. The false positive (FP) is defined as the erroneous detection of a spot as a particle, where the detected spot is actually not a particle; the false negative (FN) is defined as the erroneous detection of a spot as a non-particle, when the spot is actually a particle. Note that there is always a tradeoff between false positives and false negatives. In terms of statistical hypothesis testing, this tradeoff is a matter of balancing the risk of Type I errors (false positives
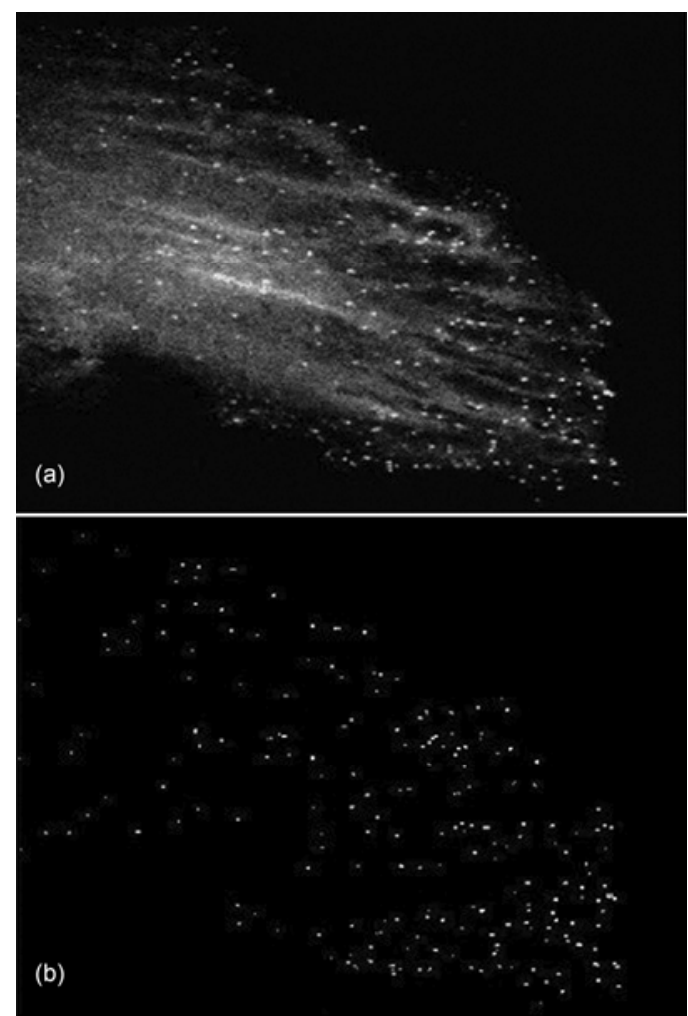

Figure 2 Particle detection of adaptor image. (a) Original image; (b) detected particles.
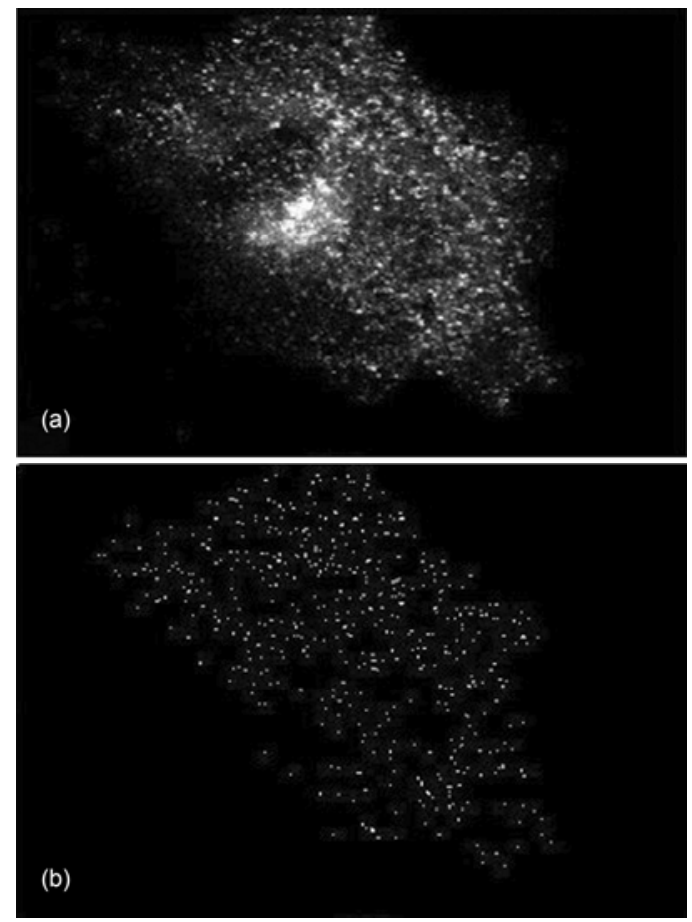

Figure 3 Particle detection of clathrin image. (a) Original image; (b) detected particles.

which reject the null hypothesis when it is true) against Type II errors (false negatives which fail to reject the null hypothesis when it is false). The true positive (TP) is defined as the true detection of a spot as a particle; the true negative (TN) is defined as the accurate detection of a spot that is indeed not a particle. In the total number of detected spots, the number of true particles is $(\mathrm{TP}+\mathrm{FN})$, and the number of non-particles is $(\mathrm{FP}+\mathrm{TN})$. In this paper, we use the true positive rate, $\mathrm{TPR}=\mathrm{TP} /(\mathrm{TP}+\mathrm{FN})$, and the false positive rate, $\mathrm{FPR}=\mathrm{FP} /(\mathrm{TP}+\mathrm{FN})$, to investigate the performance of the proposed approach. We then analyzed the adaptor and clathrin movies. Two hundred and nineteen (219) particles were counted manually in the first frame of the Adaptor movie and 637 particles were counted manually in the first frame of the Clathrin movie. In this study, a cutoff value of $\mathrm{SNR}=3$ was set empirically based on the algorithm performance. As a result, the weak particles with low contrast $(\mathrm{SNR}<3.0)$ and low total intensity (total intensity <0.5) were discarded as noise. Table 1 gives a comparison between the automated and manual detection results. It can be seen that the TPR from the adaptor and clathrin images are $98.61 \%$ and $97.65 \%$, respectively. The lower TPR for clathrin may result from the higher density of particles in the clathrin images.

To examine the validity of the proposed tracking method, we first designed a simulation. In a $100 \times 100$ image region, particles are generated randomly for ten series frames, where the velocities of the particles were normally distributed in less than two pixels for two neighboring frames. The 
Table 1 Comparison of results for automated and manual detection

\begin{tabular}{cccccc}
\hline & TPR & FPR & FP & FN & TP \\
\hline Clathrin & $97.65 \%$ & $3.13 \%$ & 20 & 15 & 622 \\
Adaptor & $98.61 \%$ & $1.39 \%$ & 3 & 3 & 216 \\
\hline
\end{tabular}

particles are merged if their distances are less than two pixels. Furthermore, only completely matched trajectories in the experiment were considered as correct. Figure 4 represents the performances of IPAN [14], 1-to-1 admission method [7], and N-to-N admission method for variable particle density. In the 1-to-1 admission method, one particle only corresponds to one particle in the next frame; while in the N-to-N method, multiple particles can correspond to one particle in the next frame, and one particle can correspond to multiple particles in the next frame. The rate of errors in particle tracking is almost the same when the particle density is lower than $10 \%$. However, the tracking error of the $\mathrm{N}$-to- $\mathrm{N}$ admission algorithm is much lower than the others when the density of particles is higher than $10 \%$. The rise in particle density leads to a high degree of splitting and merging opportunities for the particles. These results demonstrate that the $\mathrm{N}$-to- $\mathrm{N}$ admission algorithm has the advantage of detecting splitting/merging trajectories.

Figure 5 represents the analyzed life time distribution of particles for 100 frames of the adaptor images, where those particles that appeared in less than three frames are ignored as noise. It was observed that a large number of particles exist at about $18 \mathrm{~s}$. To evaluate the performance of the tracking algorithm with real image data, we calculated the tracking efficiency, which is defined by $T_{\text {traj }}=T_{\text {corr }} / T_{\text {total }}$, where $T_{\text {corr }}$ is the number of the effectively detected trajectories by our algorithm, and $T_{\text {total }}$ is the total number of the trajectories by manual tracking. Owing to the parallel optimization character, the admission algorithm with 1-to-1 correspondence is faster than traditional greedy methods

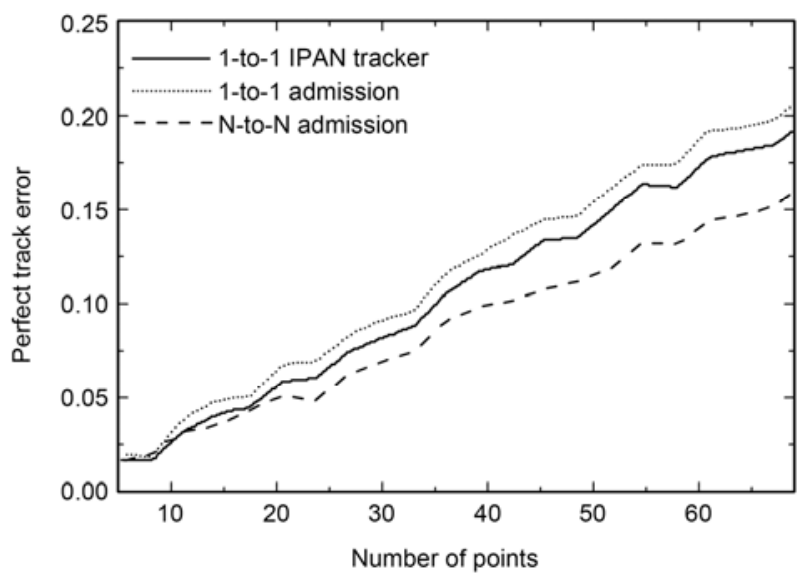

Figure 4 Variable point density performance.

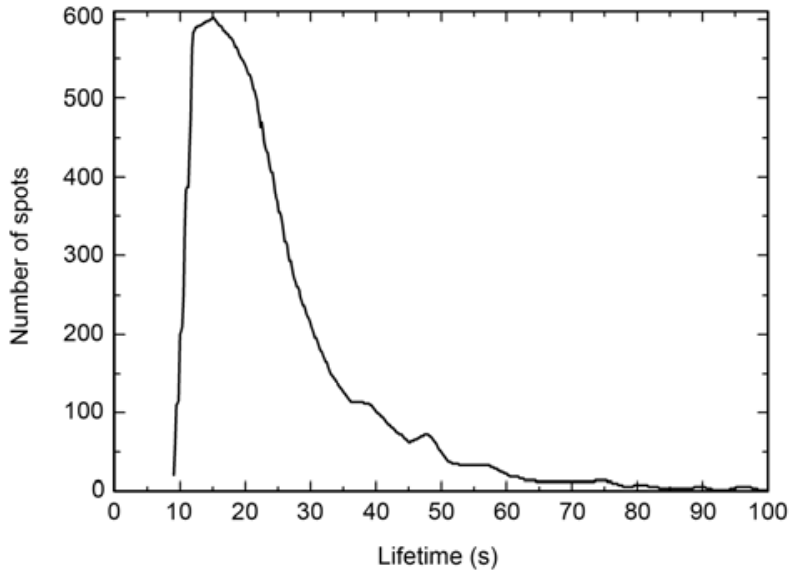

Figure 5 Life time distribution of particles.

$[9,16]$, especially for the clathrin images. When the proposed approach is extended to N-to-N correspondence, it can address the merging/splitting issue and detect 5\%-10\% more trajectories than was possible with previous methods $[9,14,16]$. Furthermore, the calculated $T_{\text {traj }}$ reaches $98.05 \%$, which can provides a better understanding of the clathrin-dependent pathway of membrane vesicular traffic.

\section{Conclusion}

When faced with the strong background noise and low contrast dynamic images of living cells, traditional segmentation methods such as single threshold, edge detection, and morphological transform do not work. In this study, we introduced an improved wavelet multi-scale product method combined with automatic threshold selection and post-processing solution to detect fast moving spots. After detection, several post-processing methods were used to segment the spots out of the circular regions and a SNR criterion was used to exclude any weak spots. For particle tracking, a new tracking method based on the greedy algorithm and stable match was proposed to extend the traditional 1-to-1 correspondence into an $\mathrm{N}$-to-N approach that can address the merging/splitting issue effectively. Results show that the proposed method worked well for the segmentation and tracking of clathrin coated pits, vessels, and receptors when applied to representative movies. The method presented here thus has the potential to provide a cost-effective solution to resolve the detection and tracking of subcellular molecular particles in living cells. The current work is focused on the optimization between two adjacent frames; the next step of this research will address global optimization and be applied to more real images.

This work was supported by the National Basic Research Program of China (2011CB707900), the National Natural Science Foundation of China (10974093, 11174141) and the Fundamental Research Funds for the Central Universities (1103020402, 1116020410 and 1112020401). 
1 Erlich M, Boll W, van Oijen A, et al. Endocytosis by random initiation and stabilization of clathrin-coated pits. Cell, 2004, 118: 591605

2 Wang Y, Lai L H. Modeling the intracellular dynamics for Vif-APO mediated HIV-1 virus infection. Chin Sci Bull, 2010, 55: 2329-2340

3 Kirchhausen T. Three ways to make a vesicle. Nat Rev Mol Cell Biol, 2000, 1: 187-198

4 Otsu N. A threshold selection method from gray-level histograms. IEEE Trans Syst Man Cybern, 1979, 9: 377-393

5 Serra P. Image Analysis and Mathematical Morphology. New York: Academic Press, 1982

6 Olivo-Marin J C. Extraction of spots in biological images using multiscale products. Pattern Recogn, 2002, 35: 1989-1996

7 Veenman C J, Marcel J T, Backer E. Resolving motion correspondence for densely moving points. IEEE Trans Pattern Anal Mach Intel, 2001, 23: 54-72

8 Eden E, Waisman D, Rudzsky M, et al. An automated method for analysis of flow characteristics of circulating particles from in vivo video microscopy. IEEE Trans Med Imag, 2005, 24: 1011-1024

9 Chetverikov D. Particle image velocimetry by feature tracking. Comput Anal Imag Patterns, 2001, 21: 325-332

10 Tvaruskó W, Bentele M, Misteli T, et al. Time-resolved analysis and visualization of dynamic processes in living cells. Proc Natl Acad Sci USA, 1999, 96: 7950-7955
11 Egmont-Petersen M, Shreiner U, Tromp S C, et al. Detection of leukocytes in contact with the vessel wall from in vivo microscope recordings using a neural network. IEEE Trans Med Imag, 2000, 47: 941-951

12 Saban M, Altinok A, Peck A, et al. Automated tracking and modeling of microtubule dynamics. In: Proceedings of the 2006 IEEE International Symposium on Biomedical Imaging: From Nano to Macro. Arlington, VA, USA, 2006. 1032-1035

13 Sethi I K, Jain R. Finding trajectories of feature points in a monocular image sequence. IEEE Trans Pattern Anal Mach Intel, 1987, 9: 56-73

14 Chetverikov D, Verestóy J. Feature point tracking for incomplete trajectories. Computing, 1998, 62: 321-338

15 Starck J L, Murtagh F, Bijaoui A. Multi-resolution support applied to image filtering and restoration. Graph Model Imag Proc, 1995, 57: $420-431$

16 Kittler J, Illingworth J. Minimum error thresholding. Pattern Recogn, 1986, 19: 41-47

17 Meijering E, Smal I, Danuser G. Tracking in molecular bioimaging. IEEE Signal Proc Mag, 2006, 23: 46-53

18 Cheezum M K, Walker W F, Guilford W H. Quantitative comparison of algorithms for tracking single fluorescent particles. Biophys J, 2001, 81: 2378-2388

19 Shaw G L, Ramachandran V S. Interpolation during apparent motion. Perception, 1982, 11: 491-494

Open Access This article is distributed under the terms of the Creative Commons Attribution License which permits any use, distribution, and reproduction in any medium, provided the original author(s) and source are credited. 